\title{
ARCHIMEDEAN VECTOR LATTICES GENERATED BY TWO ELEMENTS
}

\author{
ROGER D. BLEIER
}

\begin{abstract}
The class of vector lattices referred to in the title is investigated from the point of view of the free vector lattice on two elements. It is shown that only three of these vector lattices are indecomposable. They are then described. A complete structure theorem for projective vector lattices generated by two elements is proved. The arguments depend throughout on the precise description of the free vector lattice which is established in the first section.
\end{abstract}

There are an infinite number of (nonisomorphic) archimedean vector lattices that possess generating subsets of cardinality 2 . These include the free vector lattice FVL2 on two elements and, consequently, all projective vector lattices that can be generated by two elements.

We present here a very precise description of FVL2 and use it to catalogue, first, indecomposable archimedean vector lattices generated by two elements (we prove there are only three) and, second, projective vector lattices generated by two elements (we prove each is a cardinal sum of a finite number of the indecomposable vector lattices).

Freedom and projectivity here have their usual meanings relative to the class of all vector lattices. By the cardinal sum of vector lattices we mean the vector space direct sum with coordinatewise order. The symbol $\oplus$ will denote cardinal sum. A vector lattice is indecomposable if it cannot be written $W=A \oplus B$, where $A$ and $B$ are nonzero ideals of $W$.

The reader will no doubt find some of the proofs in this paper to be tedious. Shorter proofs (perhaps ones that generalize) would be most welcome.

1. The free vector lattice. A cone $C$ in $R^{2}$ is a subset of $R^{2}$ such that $x \in C$ and $0<r \in R$ imply $r x \in C$. $C$ is a closed cone if it is closed in the usual topology of the plane, and a convex cone if $x, y \in C$ and $0 \leqq r \leqq 1$ imply $r x+(1-r) y \in C$.

A mapping $f: R^{2} \rightarrow R$ is piecewise linear if $f$ is continuous and there exist a finite number of closed convex cones $C_{1}, \cdots, C_{t}$ in $R^{2}$ and linear functionals $f_{1}, \cdots, f_{t}: R^{2} \rightarrow R$ such that $C_{1} \cup \cdots \cup C_{t}=R^{2}$ and $f(x)=f_{i}(x)$ for all $x \in C_{i}(i=1, \cdots, t)$.

Received by the editors November 30, 1971 and, in revised form, July 6, 1972. AMS (MOS) subject classifications (1970). Primary 06A60, 06A40. 
The set of all piecewise linear maps $R^{2} \rightarrow R$ is a vector lattice under pointwise operations. We denote this vector lattice by PWL2. It was shown in [1] that the vector sublattice of PWL2 generated by the two coordinate projection maps is (up to isomorphism) the free vector lattice FVL2 on two elements. It is the purpose of this section to prove that FVL2=PWL2. We note at the outset that FVL2 does contain all the linear functionals $R^{2} \rightarrow R$.

We make several ad hoc definitions and conventions. Every cone in $R^{2}$ which contains 0 is topologically connected. We shall call a cone in $R^{2}$ connected iff $C \backslash\{0\}$ is topologically connected.

Let $f \in$ PWL2. A connected cone $C$ with nonempty interior in $R^{2}$ and with the property that there is some linear functional $f_{i}: R^{2} \rightarrow R$ such that $f(x)=f_{i}(x)$ for all $x \in C$ will be called an $f_{i}$-cone of $f$. A maximal $f_{i}$-cone of $f$ will be called an $f_{i}$-component of $f$, and also an appearance of $f_{i}$ in $f$. Each such component is a closed cone in $R^{2}$, and $R^{2}$ is the union of the components of $f$.

Only a finite number of functionals appear in $f$, and these appear only a finite number of times. If no functional appears more than once in $f$, we shall call $f$ reduced.

Let $C_{1}$ be an $f_{i}$-component of $f$ and $C_{2}$ an $f_{j}$-component of $f$ with $C_{1} \neq$ $C_{2}$. Then $f_{i}(x)=f(x)=f_{j}(x)$ for all $x \in C_{1} \cap C_{2}$, and hence $C_{1} \cap C_{2} \subseteq$ $\operatorname{Ker}\left(f_{i}-f_{j}\right)$. There are thence but three possibilities:

(i) $C_{1} \cap C_{2}=0$.

(ii) $C_{1} \cap C_{2}=\operatorname{Ker}\left(f_{i}-f_{j}\right)$, a straight line through the origin in $R^{2}$.

(iii) $C_{1} \cap C_{2}$ is a ray from the origin contained in the straight line $\operatorname{Ker}\left(f_{i}-f_{j}\right)$.

Case (i) will not be important in the sequel. We note that it holds, in particular, if $f_{i}=f_{j}$. Henceforth we shall assume $f_{i} \neq f_{j}$ if $i \neq j$.

Case (ii) holds if and only if $C_{1}$ and $C_{2}$ are half-planes. In this case either $f_{i}(x) \geqq f_{j}(x)$ for all $x \in C_{1}$, whence $f_{j}(x) \geqq f_{i}(x)$ for all $x \in C_{2}$, and $f=f_{i} \vee f_{j}$; or, $f_{i}(x) \leqq f_{j}(x)$ for all $x \in C_{1}$, whence $f_{j}(x) \leqq f_{i}(x)$ for all $x \in C_{2}$, and $f=f_{i} \wedge f_{j}$. Thus $f \in \mathrm{FVL} 2$ whenever case (ii) holds. It is important to note that this case holds if and only if $f_{i}$ and $f_{j}$ are the only functionals appearing in $f$.

In case (iii) we make some further definitions. The ray will be called an $\alpha$-boundary if $f_{i}(x) \geqq f_{j}(x)$ for all $x \in C_{1}$, or $f_{j}(x) \geqq f_{i}(x)$ for all $x \in C_{2}$. It will be called a $\beta$-boundary if $f_{i}(x) \leqq f_{j}(x)$ for all $x \in C_{1}$, or $f_{j}(x) \leqq f_{i}(x)$ for all $x \in C_{2} . C_{1} \cap C_{2}$ is then always an $\alpha$-boundary or a $\beta$-boundary, but never both.

1.1 Lemma. With the notation as above, suppose $f \in \mathrm{PWL} 2$ is reduced with exactly $k$ functionals appearing in it, and suppose $C_{2}$ is convex. If 
$C_{1} \cap C_{2}$ is an $\alpha$-boundary, then $f_{i} \wedge f$ has fewer than $k$ functionals appearing in it. If $C_{1} \cap C_{2}$ is a $\beta$-boundary, then $f_{i} \vee f$ has fewer than $k$ functionals appearing in it.

Proof. If $C_{1} \cap C_{2}$ is an $\alpha$-boundary, then $f(x)=f_{j}(x) \geqq f_{i}(x)$ for all $x \in C_{2}$. Hence $\left(f_{i} \wedge f\right)(x)=f_{i}(x)$ for all $x \in C_{2}$, and since $f$ is reduced, $f_{j}$ does not appear in $f_{i} \wedge f$. Moreover, $\left(f_{i} \wedge f\right)(x)$ is $f(x)$ or $f_{i}(x)$ for all $x \in R^{2}$, and thus fewer than $k$ functionals appear in $f_{i} \wedge f$.

The case that $C_{1} \cap C_{2}$ is a $\beta$-boundary is dual.

1.2 Lemma. Let $k \geqq 3$ be an integer. Suppose that all elements of PWL2 with fewer than $k$ functionals appearing in them are in FVL2. Let $f \in$ PWL2 be reduced with exactly $k$ functionals appearing in it. Then $f \in$ FVL2.

Proof. Let $f_{1}, \cdots, f_{k}$ be the $k$ functionals appearing in $f$. We distinguish five cases.

(a) Suppose all boundaries of $f$ are $\alpha$-boundaries. There is at most one component of $f$ which is not convex. Thus each $f_{i}$-component of $f$ shares a boundary with some convex $f_{j}$-component of $f$. By Lemma 1.1 we conclude $f_{i} \wedge f \in \mathrm{FVL} 2$ for all $i=1, \cdots, k$. Thus $f=\left(f_{1} \wedge f\right) \vee \cdots \vee\left(f_{k} \wedge f\right) \in \mathrm{FVL} 2$.

(b) Suppose all boundaries of $f$ are $\beta$-boundaries. The dual argument shows $f \in$ FVL2.

(c) Suppose there is a component of $f$, say the $f_{i}$-component, which shares an $\alpha$-boundary with some convex component of $f$ and a $\beta$-boundary with some other convex component of $f$. Then by Lemma 1.1 we have $f_{i} \wedge f \in \mathrm{FVL} 2$ and $f_{i} \vee f \in \mathrm{FVL} 2$. Thus $f=\left(f_{i} \wedge f\right)+\left(f_{i} \vee f\right)-f_{i} \in \mathrm{FVL} 2$.

(d) Suppose some component, for definiteness the $f_{k}$-component, is not convex and has two $\alpha$-boundaries, while all other boundaries are $\beta$ boundaries. Designate the components adjacent to the $f_{k}$-component as the $f_{i}$ - and $f_{j}$-components. From the fact that both boundaries of the $f_{k}$ component are $\alpha$-boundaries, we conclude that the only functionals appearing in $f_{i} \wedge f_{j} \wedge f$ are $f_{1}, f_{2}, \cdots, f_{k-1}$. Thus $f_{i} \wedge f_{j} \wedge f \in$ FVL2. By Lemma $1.1, f_{i} \vee f \in \mathrm{FVL} 2$ since the $f_{i}$-component shares a $\beta$-boundary with some convex component. Similarly, $f_{j} \vee f \in$ FVL2. Thus $\left(f_{i} \wedge f_{j}\right) \vee f=\left(f_{i} \vee f\right) \wedge$ $\left(f_{j} \vee f\right) \in \mathrm{FVL} 2$, and hence $f=\left(\left(f_{i} \wedge f_{j}\right) \wedge f\right)+\left(\left(f_{i} \wedge f_{j}\right) \vee f\right)-\left(f_{i} \wedge f_{j}\right) \in \mathrm{FVL} 2$.

(e) The dual of (d).

Finally we prove that cases (a)-(e) exhaust all possibilities. Suppose $f$ is not included in any of cases (a), (b), (d), or (e). Then $f$ has at least one $\alpha$ boundary and at least one $\beta$-boundary. Thus some $f_{i}$-component of $f$ has both an $\alpha$-boundary and a $\beta$-boundary. If the components adjacent to the $f_{i}$-component are both convex, then (c) is satisfied. Thus we can assume that $f$ has a nonconvex component. If the nonconvex component has both an $\alpha$ - and a $\beta$-boundary, then (c) is satisfied. Suppose now that both 
boundaries of the nonconvex component are $\alpha$-boundaries. Since $f$ is not included in case (d), there is some other $\alpha$-boundary. There is then some $f_{j}$-component of $f$ with an $\alpha$-boundary and a $\beta$-boundary for which case (c) applies. The dual argument applies if both boundaries of the nonconvex component are $\beta$-boundaries.

\subsection{TheOREM. FVL2 $=$ PWL2.}

Proof. Let $f \in \mathrm{PWL} 2$, and let $k$ be the number of functionals appearing in $f$. We show $f \in \mathrm{FVL} 2$ by using induction on $k$. If $k=1$, then $f$ is linear, and hence $f \in \mathrm{FVL} 2$. The case $k=2$ has been handled previously. So suppose $k \geqq 3$ and that all members of PWL2 with fewer than $k$ functionals appearing in them are members of FVL2.

With $k$ fixed, we perform a second induction, this time on the number $r$ of functionals appearing more than once in $f$. Note $0 \leqq r \leqq k$. If $r=0$, then $f$ is reduced, and by Lemma 1.2 we have $f \in$ FVL2. Suppose now $r \geqq 1$, and let $f_{1}, \cdots, f_{r}$ denote those functionals appearing more than once in $f$, and $f_{r+1}, \cdots, f_{k}$ the remaining functionals appearing in $f$. Moreover, assume $f_{1}$ is the zero-functional if the zero-functional appears more than once in $f$.

Let $C$ be the union of the $f_{1}$-components of $f$, and let $S_{1}, \cdots, S_{t}$ be the topological components of $R^{2} \backslash C$. For each $i=1, \cdots, t$ define $g_{i}: R^{2} \rightarrow R$ by

$$
\begin{aligned}
g_{i}(x) & =f(x)-f_{1}(x) & & \text { if } x \in S_{i}, \\
& =0 & & \text { if } x \in R^{2} \mid S_{i} .
\end{aligned}
$$

Note that $g_{i} \in$ PWL2. The only functionals which can appear in $g_{i}$ are $0, f_{2}-f_{1}, \cdots, f_{k}-f_{1}$, and the only functionals which can appear more than once are $f_{2}-f_{1}, \cdots, f_{r}-f_{1}$. (Note that the zero-functional appears only once.) Hence the number of functionals appearing in $g_{i}$ is $\leqq k$, and the number of functionals appearing more than once in $g_{i}$ is $\langle r$. If fewer than $k$ functionals appear in $g_{i}$, then by the induction hypothesis on $k$ we have $g_{i} \in \mathrm{FVL} 2$. If exactly $k$ functionals appear in $g_{i}$, then by the induction hypothesis on $r$ we have $g_{i} \in \mathrm{FVL} 2$.

Thus $g_{i} \in$ FVL2 for all $i=1, \cdots, t$. Since $f=g_{1}+\cdots+g_{t}+f_{1}$, we conclude $f \in$ FVL2. This completes the proof.

2. Indecomposable vector lattices. In this section we will investigate those archimedean quotients of FVL2 which are indecomposable. The main result is that there are (up to isomorphism) only three of them, and they can be easily described. Our technique will combine some elementary maximal ideal theory with the theorem of the previous section.

If $0 \neq x \in R^{2}$ we let $\bar{x}=\{r x \mid 0 \leqq r \in R\}$, the ray from the origin through $x$. Define $\varphi_{x}$ : FVL2 $\rightarrow R$ by $\varphi_{x}(f)=f(x)$. Then $\operatorname{Ker} \varphi_{x}$ is a maximal ideal of FVL2, to be denoted $M_{x}$. If $f \in \mathrm{FVL} 2$ then $f(r x)=r f(x)$ for all $x \in R^{2}$ and 
$0 \leqq r \in R$. (In particular, $f(0)=0$.) It follows then from the compactness of the unit circle that each maximal ideal of FVL2 is of the form $M_{x}$ for some $x \in R^{2}$. Moreover, $M_{x}=M_{y}$ if and only if $\bar{x}=\bar{y}$.

If $f \in \mathrm{FVL} 2$ and $A$ is an ideal of FVL2, we define $T(f)=\left\{x \in R^{2} \mid f(x) \neq 0\right\}$ and $T(A)=\bigcup\{T(f) \mid f \in A\} . T(f)$ and $T(A)$ are then open cones in $R^{2}$. Note $T\left(M_{x}\right)=R^{2} \backslash \bar{x}$.

The foregoing notation will be used throughout the sequel.

2.1 Lemma. Suppose $A$ is an ideal of FVL2 such that FVL2/A is archimedean. If $T(f) \subseteq T(A)$ then $f \in A$.

Proof. The intersection of the maximal ideals of FVL2/ $A$ is 0 since FVL2 (and hence FVL2/ $A$ ) has a strong unit and FVL2/ $A$ is archimedean [5]. Thus $A$ is an intersection of maximal ideals of FVL2. Hence $A=$ $\bigcap\left\{M_{x} \mid x \in X\right\}$ for some subset $X$ of $R^{2}$. Suppose $T(f) \subseteq T(A)$. Then $T(f) \subseteq T\left(M_{x}\right)=R^{2} \backslash \bar{x}$, whence $f(x)=0$ and $f \in M_{x}$ (all $\left.x \in X\right)$. Thus $f \in A$.

2.2 Lemma. Suppose FVL2/A is archimedean and let $C=R^{2} \backslash(T(A) \cup\{0\})$. Then FVL2/A is isomorphic to the vector lattice $\left\{\left.f\right|_{C} \mid f \in \mathrm{FVL2}\right\}$ of restrictions of the elements of FVL2 to $C$. (If $C=\varnothing$, take $\left.\left.f\right|_{C}=0.\right)$

Proof. The map restricting $f \in \mathrm{FVL} 2$ to $\left.f\right|_{C}$ is a vector lattice homomorphism whose kernel, by Lemma 2.1 , is $A$.

2.3 Lemma. Suppose FVL2/A is archimedean. Then FVL2/A is indecomposable if and only if $T(A)$ is connected.

Proof. Let $C=R^{2} \backslash(T(A) \cup\{0\})$, and $W=\left\{\left.f\right|_{C} \mid f \in \mathrm{FVL} 2\right\}$. By the preceding lemma, $W$ is isomorphic to FVL2/ $A$.

If $T(A)$ is connected, then $C$ is connected, and since $\left\{x \in R^{2} \mid g(x) \neq 0\right.$ for some $g \in W\}=C, W$ is indecomposable.

Conversely, suppose $T(A)$ is not connected. Then $T(A)$ has at least two topological components $S_{1}$ and $S_{2} . S_{1}$ and $S_{2}$ are each cones in $R^{2}$, and since $T(A)$ is an open subset of the locally connected space $R^{2}$, they are also open subsets of $R^{2}$. $R^{2} \backslash\left(S_{1} \cup S_{2} \cup\{0\}\right)$ has precisely two topological components $D_{1}$ and $D_{2}$. Let $J_{1}=\left\{g \in W \mid g(x)=0\right.$ for all $\left.x \in C \cap D_{2}\right\}$, and $J_{2}=\left\{g \in W \mid g(x)=0\right.$ for all $\left.x \in C \cap D_{1}\right\}$.

$J_{1}$ and $J_{2}$ are nonzero ideals in $W$ and $J_{1} \cap J_{2}=0$. Let $g \in W$. Then $g=$ $\left.f\right|_{C}$ for some $f \in$ FVL2. It is easy to construct a piecewise linear map $f_{1}: R^{2} \rightarrow R$ such that $f_{1}(x)=f(x)$ for all $x \in D_{1}$ and $f_{1}(x)=0$ for all $x \in D_{2}$. Similarly, there exists a piecewise linear map $f_{2}: R^{2} \rightarrow R$ such that $f_{2}(x)=$ $f(x)$ for all $x \in D_{2}$ and $f_{2}(x)=0$ for all $x \in D_{1}$. By Theorem 1.3 we have $f_{1}, f_{2} \in$ FVL2. Let $g_{i}=\left.f_{i}\right|_{C}, i=1,2$. Then $g_{i} \in J_{i}$, and $g=g_{1}+g_{2}$. Thus $W=$ $J_{1} \oplus J_{2}$, and the proof is complete. 
2.4 THEOREM. There are (up to isomorphism) only three nonzero indecomposable archimedean vector lattices which have a generating subset of cardinality 2.

Proof. Suppose $W_{1}$ and $W_{2}$ each are archimedean vector lattices with two element generating subsets, and that neither is free nor a copy of $R$. We show $W_{1}$ is isomorphic to $W_{2}$.

For $i=1,2$, there is an ideal $A_{i}$ such that $W_{i}$ is isomorphic to FVL2/ $A_{i}$. Let $C_{i}=R^{2} \backslash\left(T\left(A_{i}\right) \cup\{0\}\right)$. Then without loss of generality we can assume $W_{i}=\left\{\left.f\right|_{C_{i}} \mid f \in \mathrm{FVL} 2\right\}$.

$T\left(A_{i}\right), i=1,2$, is an open cone in $R^{2}$, which by Lemma 2.3 is connected. If $T\left(A_{i}\right)=\varnothing$, then $A_{i}=0$ and $W_{i}$ is free. Thus $T\left(A_{i}\right) \neq \varnothing$. If $T\left(A_{i}\right)=R^{2} \backslash\{0\}$, then by Lemma 2.2, $W_{i}=0$. Thus $T\left(A_{i}\right) \neq R^{2} \backslash\{0\}$. Finally, if $R^{2} \backslash T\left(A_{i}\right)$ is a ray from the origin, then $A_{i}$ is maximal and so $W_{i} \cong R$. Thus the cone $R^{2} \backslash T\left(A_{i}\right)$ has interior, and hence the topological closure of $T\left(A_{i}\right)$ is properly contained in $R^{2}$.

Let $L_{i}$ be the line through the origin which bisects $T\left(A_{i}\right)$. This splits $T\left(A_{i}\right)$ into two convex cones $D_{i}$ and $E_{i}$. Choose vectors $a_{i}, b_{i}$, and $c_{i}$ such that $\overline{a_{i}}$ and $\bar{b}_{i}$ are the boundaries of $D_{i}$, and $\bar{b}_{i}$ and $\bar{c}_{i}$ are the boundaries of $E_{i}$. By the considerations of the preceding paragraph, $a_{i}$ and $b_{i}$ are linearly independent (for fixed $i=1,2$ ) and so are $b_{i}$ and $c_{i}$. Let $g: R^{2} \rightarrow R^{2}$ be the vector space isomorphism such that $g\left(a_{1}\right)=a_{2}$ and $g\left(b_{1}\right)=b_{2}$. Then $g\left(D_{1}\right)=$ $D_{2}$. Similarly, letting $h: R^{2} \rightarrow R^{2}$ be the vector space isomorphism such that $h\left(b_{1}\right)=b_{2}$ and $h\left(c_{1}\right)=c_{2}$, we have $h\left(E_{1}\right)=E_{2}$.

Now let $G$ be the closed half-plane bounded by $L_{1}$ which contains $D_{1}$, and let $H$ be the closed half-plane bounded by $L_{1}$ which contains $E_{1}$, and define $\alpha: R^{2} \rightarrow R^{2}$ by

$$
\begin{aligned}
\alpha(x)=g(x) & \text { if } x \in G, \\
=h(x) & \text { if } x \in H .
\end{aligned}
$$

Then $\alpha$ is a well-defined bijection of $R^{2}$ to $R^{2}$, and $\alpha\left(D_{1}\right)=D_{2}$ and $\alpha\left(E_{1}\right)=$ $E_{2}$.

Note that both $f \circ \alpha$ and $f \circ \alpha^{-1}$ are piecewise linear whenever $f: R^{2} \rightarrow R$ is. Using Theorem 1.3 we define $\alpha^{*}:$ FVL2 $\rightarrow$ FVL2 by $\alpha^{*}(f)=f \circ \alpha^{-1}$. It is easily verified that $\alpha^{*}$ is a vector lattice automorphism. Thus FVL2/ $A_{1}$ is isomorphic to FVL2/ $\alpha^{*} A_{1}$; in particular, FVL2/ $\alpha^{*} A_{1}$ is archimedean.

Now $T\left(\alpha^{*} A_{1}\right)=\alpha\left(T\left(A_{1}\right)\right)=\alpha\left(D_{1} \cup E_{1}\right)=\alpha\left(D_{1}\right) \cup \alpha\left(E_{1}\right)=D_{2} \cup E_{2}=T\left(A_{2}\right)$. Thus by Lemma 2.1 ,

$\alpha^{*} A_{1}=\left\{f \in \mathrm{FVL} 2 \mid T(f) \subseteq T\left(\alpha^{*} A_{1}\right)\right\}=\left\{f \in \mathrm{FVL} 2 \mid T(f) \subseteq T\left(A_{2}\right)\right\}=A_{2}$.

Hence FVL2 $/ A_{1}$ is isomorphic to FVL2/ $A_{2}$, and thus $W_{1}$ is isomorphic to $W_{2}$. 
FVL2 and $R$ are both archimedean and indecomposable. Let $E$ be the vector sublattice of the vector lattice of functions from $R$ to $R$ (pointwise operations) generated by the function $f(x)=x$ and the constant function 1 . $E$ is archimedean, and since $R$ is connected, $E$ is indecomposable. It was shown in [3] that $E$ is not isomorphic to FVL2. Clearly, $E$ is not isomorphic to $R$. Thus $E, \mathrm{FVL2}$, and $R$ are the three nonisomorphic (nonzero) archimedean indecomposable vector lattices which can be generated by two elements.

The proof of the theorem shows also that $E$ is isomorphic to the vector lattice of restrictions of the elements of FVL2 to $C$, where $C$ is any connected closed cone properly contained in $R^{2}$ and having nonempty interior.

It seems desirable to give a list of algebraic properties by which $E, R$, and FVL2 can be distinguished from each other abstractly. Proofs will be omitted although for the case of FVL2 many of them appear in [3].

(1) $R$ is totally ordered. $E$ and FVL2 are not.

(2) $R$ has basic elements. $E$ and FVL2 do not.

(3) $E$ has a nonzero proper ideal which is invariant under all automorphisms. $R$ and FVL2 do not.

(4) There is an automorphism of FVL2 which leaves no maximal ideal of FVL2 invariant. $E$ and $R$ do not have this property.

(5) FVL2 is distinguished from all other archimedean vector lattices generated by two elements (including $E$ and $R$ ) by the fact that no pair of positive elements generate FVL2.

3. Projective vector lattices. FVL2 is a projective vector lattice since it is free. The free vector lattice on 1 element is the cardinal sum of two copies of $R$. Thus $R$ is a projective vector lattice. Finally, it was proved in [3] that the vector lattice $E$ of the preceding section is a projective vector lattice. Thus each of the indecomposable archimedean vector lattices generated by two elements is a projective vector lattice.

A description of finitely generated projective vector lattices has been given by K. Baker [1]. We state his theorem here for the case of two generators.

3.1 LeMMA (BAKER [1]). FVL2/A is a projective vector lattice if and only if $A$ is a principal ideal of FVL2.

We note that if $A$ is the principal ideal generated by $h \in \mathrm{FVL} 2$ then $T(A)=T(h)$ has but a finite number of topological components and each is an open cone in $R^{2}$.

3.2 THEOREM. Let $W$ be a vector lattice which has a generating subset of cardinality 2. $W$ is projective if and only if $W$ is the cardinal sum of a finite number of indecomposable archimedean vector lattices. 
Proof. Suppose $W$ is the cardinal sum of the indecomposable archimedean vector lattices $P_{1}, \cdots, P_{n}$. Since $W$ is generated by two elements, so is each of its cardinal summands. Thus $P_{1}, \cdots, P_{n}$ are projective vector lattices. By freedom there exists a vector lattice epimorphism $\sigma:$ FVL2 $\rightarrow$ $P_{1} \oplus \cdots \oplus P_{n}$. Let $\tau_{i}: P_{1} \oplus \cdots \oplus P_{n} \rightarrow P_{i}$ be the $i$ th projection. Since $P_{i}$ is projective, $\operatorname{Ker}\left(\tau_{i} \circ \sigma\right)$ is a principal ideal by Lemma 3.1. Thus $\bigcap_{i=1}^{n} \operatorname{Ker}\left(\tau_{i} \circ \sigma\right)$ is a principal ideal of FVL2. Furthermore, $\operatorname{Ker} \sigma=$ $\bigcap_{i=1}^{n} \operatorname{Ker}\left(\tau_{i} \circ \sigma\right)$. Thus, again by Lemma $3.1, \operatorname{Im} \sigma=W$ is a projective vector lattice.

Conversely, suppose $W$ is projective. Then $W$ is isomorphic to FVL2/A for some principal ideal $A$ of FVL2. Let $C=R^{2} \backslash(T(A) \cup\{0\})$. Then $C$ has only a finite number of topological components $K_{1}, \cdots, K_{r}$.

By Lemma 2.2 we can assume without loss of generality that $W=$ $\left\{\left.f\right|_{C} \mid f \in\right.$ FVL2 $\}$. Let $J_{i}=\left\{g \in W \mid g(x)=0\right.$ for all $\left.x \in R^{2} \mid K_{i}\right\}$. Then $J_{i}$ is an ideal of $W$, and since $\left\{x \in R^{2} \mid g(x) \neq 0\right.$ for some $\left.g \in J_{i}\right\}=K_{i}$ is connected, $J_{i}$ is indecomposable. Also, $J_{1}, \cdots, J_{r}$ are pairwise disjoint.

Let $g \in W$. For each $i=1, \cdots, r$ construct $f_{i} \in$ PWL2 such that $f_{i}(x)=$ $g(x)$ for all $x \in K_{i}$ and $f_{i}(x)=0$ for all $x \in C \backslash K_{i}$. By Theorem 1.3 we have $f_{i} \in \mathrm{FVL2}$. Let $g_{i}=\left.f_{i}\right|_{C}$. Then $g=g_{1}+\cdots+g_{r} \in J_{1} \oplus \cdots \oplus J_{r}$. Thus $W=$ $J_{1} \oplus \cdots \oplus J_{r}$ and the proof is complete.

REMARK. It is a corollary to Baker's original theorem that the cardinal sum of any finite number of finitely generated projective vector lattices is again a projective vector lattice. The argument is that used in the first half of the above proof. An alternative proof of this fact that does not rely on Baker's theorem has recently been given by Jorge Martinez. It is known that the cardinal sum of an uncountable collection of projective vector lattices is never projective.

If $A$ is a nonzero ideal of FVL2, then FVL2/ $A$ is not isomorphic to FVL2 [3, Theorem 5.2]. Thus if the summand $J_{i}$ in the proof of Theorem 3.2 is a copy of FVL2, then the remaining summands of $W$ must be 0 .

Now, let $n, m$ be nonnegative integers such that $n+m \geqq 1$. The ideal $A$ in Theorem 3.2 can be so chosen that $C=R^{2} \backslash(T(A) \cup\{0\})$ has $n+m$ topological components, $n$ of which have nonempty interior and $m$ of which (rays) do not. Referring to the proof of Theorem 3.2, if $K_{i}$ is a single ray then $J_{i} \cong R$, and if $K_{i}$ has interior, but $K_{i} \neq R^{2} \backslash\{0\}$, then $J_{i} \cong E\left(J_{i} \neq \mathrm{FVL} 2\right.$

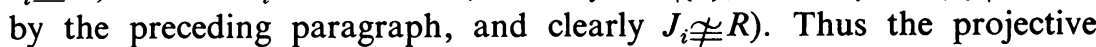
vector lattice FVL2/ $A$ is the cardinal sum of $n$ copies of $E$ and $m$ copies of $R$.

Conversely, if $A$ is any nonzero principal ideal of FVL2, then for some nonnegative integers $n$ and $m, C=R^{2} \backslash(T(A) \cup\{0\})$ has $n+m \geqq 1$ components, $n$ of which have nonempty interior and $m$ of which are rays, and the considerations above apply. 
To summarize, we have given a complete and independent system of numerical invariants for the structure of projective vector lattices generated by two elements. We formalize this in the following theorem.

3.3 THEOREM. The vector lattice $W$ is a projective vector lattice with a generating subset of cardinality 2 if and only if $W$ is isomorphic to FVL2 or $W$ is the cardinal sum of $n$ copies of $E$ and $m$ copies of $R$ (where $n, m$ are arbitrary nonnegative integers).

\section{REFERENCES}

1. K. Baker, Free vector lattices, Canad. J. Math. 20 (1968), 58-66. MR 37 \#123.

2. G. Birkhoff, Lattice theory, Amer. Math. Soc. Colloq. Publ., vol. 25, Amer. Math. Soc., Providence, R.I., 1967. MR 37 \#2638.

3. R. Bleier, Free vector lattices, Trans. Amer. Math. Soc. 176 (1973), 73-87.

4. P. Conrad, Lattice ordered groups, Tulane University, New Orleans, La., 1970.

5. K. Yosida, On a vector lattice with unit, Proc. Japan Acad. 17 (1941), 121-124. MR 3, 210.

Department of Mathematics, University of Kansas, Lawrence, Kansas 66044

Current address: Department of Mathematics, University of Texas, Austin, Texas 78712 